\title{
Capacidad explicativa de un modelo de competencias socioemocionales asociadas al uso de drogas en jóvenes de educación media básica
}

\author{
David Bruno Díaz-Negrete', Jessica Zapata-Téllez', P. Isaías Castillo-Franco', Lourdes María López-Granados', \\ Alberto J. Córdova-Alcaráz', Carmen Fernández-Cáceres ${ }^{1}$ \\ I Centros de Integración Juvenil, A.C., México
}

\section{RESUMEN}

Introducción: las competencias socioemocionales integran conocimientos, actitudes y habilidades para el manejo de afectos, entablar relaciones interpersonales y tomar decisiones responsables, entre otros. El presente trabajo tuvo como objetivo estimar la capacidad explicativa de un modelo de competencias socioemocionales respecto al consumo de drogas en estudiantes de educación secundaria. Método: estudio ex post facto, con comparación de una muestra de 512 estudiantes distribuidos en dos grupos: usuarios y no usuarios de drogas alguna vez en la vida. Se aplicó un cuestionario que incluyó escalas de inadecuada regulación emocional, bajo control conductual, toma irreflexiva de decisiones, dificultad para el establecimiento de relaciones positivas con pares y para el ajuste escolar. Se analizaron diferencias de medias y se aplicó un modelo de regresión logística, incluyendo estas variables como predictores del uso de drogas. Resultados: se identificaron como predictores significativos del uso experimental de drogas, una inadecuada regulación emocional, un deficiente ajuste escolar y un bajo control conductual. Discusión: los hallazgos obtenidos corroboran la relación existente entre competencias socioemocionales deficitarias y uso de sustancias reportado en otros estudios y proporcionan una base empírica para el desarrollo de programas preventivos dirigidos al medio escolar.

Palabras clave: uso de drogas, regulación emocional, control conductual, ajuste escolar, estudiantes.

\begin{abstract}
Introduction: the socio-emotional competences integrate knowledge, attitudes and abilities for the handling of affections, establish interpersonal relationships, and take responsible decisions, among others. The objective of this study was to estimate the explanatory power of a social-emotional competency model regarding drug use in junior high school students. Method: ex post facto study, with a comparison sample of 512 students, separated in two groups: lifetime users and non-users. A questionnaire was applied that included scales of inadequate emotional regulation, low behavioral control, thoughtless decision making, difficulty in establishing positive relationships with peers and for school adjustment. Differences in means were analyzed and a logistic regression model was applied, including these variables as predictors for drug use. Results: inadequate emotional regulation, poor school adjustment and low behavioral control were identified as significant predictors of experimental drug use. Discussion: the findings corroborate the existing relationship between a social-emotional competencies deficit and substance use reported in other studies and provides an empirical basis for the development of preventive programs aimed to the school environment.
\end{abstract}

Keywords: drug use, emotional regulation, behavioral control, school adjustment, students.

\footnotetext{
Autor de correspondencia:

D. Bruno Díaz Negrete. Avenida San Jerónimo núm. 372, col. Jardines del Pedregal, alcaldía de Álvaro Obregón, Ciudad de México, C.P. 01900. Tel.: (0155) 59994949 ext. 1910.

Recibido: 16 de agosto de 2018

Aprobado: 5 de noviembre de 2018

doi: 10.28931/riiad.2018.2.04
} 


\section{INTRODUCCIÓN}

Según la Encuesta Nacional de Consumo de Drogas en Estudiantes (Instituto Nacional de Psiquiatría, Comisión Nacional contra las Adicciones y Secretaría de Salud, 2015), $12.5 \%$ de la población estudiantil de nivel medio ha utilizado drogas ilegales alguna vez en la vida. Esto pone de manifiesto la necesidad de desarrollar programas preventivos basados en la evidencia que garanticen una más efectiva atención del problema en el contexto escolar. Uno de los enfoques que en los años recientes ha cobrado relevancia en el diseño de programas dirigidos a los jóvenes es el relativo al desarrollo de competencias socioemocionales (Collaborative for Academic, Social, and Emotional Learning [CASEL], 2015; Lerner, 2004; Lerner, Phelps, Forman, \& Bowers, 2009; Secretaría de Educación Pública [SEP] y Programa de las Naciones Unidas para el Desarrollo [PNUD] (SEP/ PNUD, 2018). Estos programas han sido originalmente impulsados por organizaciones internacionales de inclinación neoliberal, respondiendo a políticas públicas de este corte en el campo de la educación (Díaz, 2006); no obstante, pueden conformar un aporte significativo a la resolución de las necesidades existentes en materia de prevención del uso de drogas entre los jóvenes de países como México.

Las competencias socioemocionales se han entendido como el conjunto de conocimientos, habilidades psicológicas, sensoriales y motoras, así como comportamientos y disposiciones sociales, necesarios para encarar con eficacia una actividad o tarea en el contexto de situaciones concretas (Jonnaert, Barrette, Masciotra, \& Yaya, 2006). Se trata, así, de una combinación de información, habilidades, actitudes y conductas, disponible para ser puesta en acción en situaciones inéditas (Díaz, 2006). Entre los distintos modelos teóricos relacionados con competencias socioemocionales se pueden destacar el de las Cinco C de Lerner: competencia, conexión, confianza, cuidado y carácter (Lerner, 2004; Lerner et al., 2009; Travis \& Leech, 2014), y el del programa CoIlaborative for Academic, Social and Emotional Learning, consistente igualmente en cinco dominios de competencia que comprenden habilidades relacionales, toma de decisiones responsable, autocontrol, autoconciencia y conciencia social (CASEL, 2015).

Como antecedente de este enfoque, debe señalarse el trabajo de H. Gardner, quien plantea entender la inteligencia como un conjunto de capacidades diferenciadas, estructuradas de manera reticular. Los grupos autónomos de competencias o "inteligencias múltiples" descritos por Gardner son: inteligencia lingüística-verbal, musical, lógica-matemática, espacial, corporal-cinestésica, intrapersonal, interpersonal y naturalista (Gardner, 1983).
Por su parte, en 1990, en su trabajo sobre "inteligencia emocional", Salovey y Mayer (1990) Ilamaron la atención respecto a la importancia de las emociones y de su manejo en la vida cotidiana. Más tarde, D. Goleman (2001) recurrió al mismo término de "inteligencia emocional", entendido como un conjunto de habilidades entre las que destacan el autocontrol, la automotivación y la perseverancia; así como el control de impulsos, el diferimiento de la gratificación, la regulación del estado de ánimo y la capacidad de empatizar y confiar en los demás.

Por su parte, Bar-On (2006) definió la inteligencia emocional como la capacidad de entender y regular las propias emociones y su expresión, a fin de lograr un mejor ajuste psicológico y social, y propuso un modelo con cinco componentes: intrapersonal (autoconciencia, independencia y autoconcepto), interpersonal (empatía, responsabilidad social y manejo de las relaciones interpersonales), manejo del estrés (tolerancia y control de impulsos), estado de ánimo (búsqueda y mantenimiento de la felicidad) y adaptación/ajuste (estrategias de solución de problemas, prueba de realidad y flexibilidad). Este modelo se instrumentó en el Emotional Quotient Inventory (EQ-i), del cual existen distintas versiones en español (López-Zafra, Pulido, \& Berrios, 2014).

En cuanto a los factores de interés en el presente estudio, la investigación indica, en primer lugar, que la capacidad para regular las propias emociones tiene un impacto protector contra el uso de tabaco, alcohol y otras sustancias. En particular, se ha encontrado que la probabilidad de usar drogas se asocia con un déficit en el reconocimiento y la regulación de emociones, y que, ante situaciones o eventos afectivamente perturbadores, los adolescentes que no cuentan con estrategias para comprender y sobreponerse a sus sentimientos negativos corren un mayor riesgo de consumir sustancias (Hessler \& Fainsilber, 2010; Hill \& Maggi, 2011; Sharma, Gulati, \& Misra, 2017). Claros y Sharma (2010) reportaron que la inteligencia emocional se asocia significativamente con el uso de alcohol, para el caso de regulación y manejo de emociones, y con el de mariguana, para el caso de percepción, regulación y manejo de emociones. De modo similar, Fernández-Serrano, Moreno-López, Pérez-García, y Verdejo-García (2012) mostraron que pacientes dependientes de cocaína presentaban calificaciones de inteligencia emocional significativamente más bajas que controles sanos. Por su parte, Law y Yan (2011) sostienen que el fortalecimiento de competencias emocionales contribuye de manera representativa a reducir el riesgo de utilizar sustancias, en tanto que Ferreira, Simoes, Gaspar, Ramiro, y Alves (2012) encontraron que distintas competencias socio-emocionales (empatía, cooperación, comunicación, establecimiento de 
metas y aspiraciones) tienen una relación inversamente proporcional con el uso de drogas en adolescentes.

Por otro lado, se ha identificado el vínculo con pares involucrados en el uso de drogas y en actividades antisociales como un factor de alto riesgo para el consumo de drogas (Peñafiel, 2009; Rodríguez, Pérez, \& Córdova-Alcaráz, 2007); también se ha reportado la inexistencia de diferencias significativas entre consumidores y no consumidores a este respecto (Pérez-Islas \& Arellanez-Hernández, 2015). Por el contrario, Manrique-Abril, Ospina, y García-Ubaque (2009) subrayan la influencia favorable que, sobre los adolescentes, pueden ejercer compañeros miembros de colectivos deportivos o recreacionales positivamente orientados.

Asimismo, diversos estudios han demostrado que la impulsividad y el bajo control de impulsos constituyen un factor altamente relacionado con el consumo de sustancias y la recaída después de que éste ha sido suspendido (De Witt, 2009; Díaz \& García-Aurrecoechea, 2008; Díaz \& Moral, 2018; Pedrero, 2007; Perea-Baena \& Oña-Compan, 2011; Wills, Pokhrel, Morehouse, \& Fenster, 2011). En un sentido similar, los hallazgos de la investigación resaltan el papel de una inadecuada toma de decisiones no solamente en cuanto al uso de drogas, sino también en otros diversos comportamientos de riesgo (Blair et al., 2018; Carpenter, Bedi, \& Vadhan, 2015; Ekhtiari, Víctor, \& Paulus, 2016; Mata et al., 2011; Sussman et al., 2016).

Por último, desde muy temprano se ha identificado el ajuste escolar como factor protector contra el consumo de sustancias (Kandel, 1985; Smart, Medina-Mora, Terraoba, \& Varma, 1981; Smith \& Fogg, 1978). Hawkings, Catalano, y Miller (1992) señalan que los estudiantes que muestran poco interés en la escuela o en el logro académico presentan mayor riesgo de manifestar problemas de conducta diversos. Asimismo, un menor rendimiento académico se ha asociado con una mayor probabilidad de consumo de tabaco (Arillo-Santillán et al., 2002) y alcohol (Villarreal-González, Sánchez-Sosa, Musitu, \& Varela, 2010) entre los adolescentes. A la inversa, se ha mostrado que una mayor capacidad de ajuste escolar se asocia con un menor riesgo de utilizar drogas ilegales (ClJ, 1999; Díaz \& García-Aurrecoechea, 2008) y que su fortalecimiento como factor protector puede constituir un componente sustantivo de la prevención del problema en el contexto escolar (ClJ, 2015).

A partir de estos antecedentes, Centros de Integración Juvenil ha propuesto el desarrollo y la aplicación de un programa de prevención escolar del uso de drogas dirigido a estudiantes de educación media, basado en un modelo de competencias socioemocionales que incluye el fortalecimiento de factores como la regulación emocional, el establecimiento de relaciones positivas con pares, el control conductual, la toma reflexiva y responsable de decisiones y la capacidad de responder satisfactoriamente a las demandas de ajuste del medio escolar (CIJ, 2016, 2017). El presente estudio persigue ofrecer una base empíricamente sustentada al programa propuesto, a partir de dar cuenta de la capacidad predictiva que tienen dichas competencias socioemocionales del uso experimental de drogas.

\section{MÉTODO}

\section{Diseño}

Se desarrolló un estudio con diseño ex post facto, transversal, con dos grupos de comparación de estudiantes usuarios y no usuarios de drogas ilícitas.

\section{Muestra}

La muestra fue extraída de una muestra original, no probabilística, integrada por 3,050 estudiantes de primer grado de educación secundaria, captados en seis escuelas del sistema público de educación del área metropolitana de la Ciudad de México. De dicha muestra se seleccionó, primero, a los estudiantes que refirieron haber consumido algún tipo de droga ilegal alguna vez en la vida (mariguana, inhalables, cocaína u otras); resultó una submuestra de 256 estudiantes. Posteriormente, para la conformación del grupo de comparación, se hizo una selección aleatoria de 256 estudiantes más, extraídos de los 2,756 casos que refirieron nunca haber consumido drogas ilegales. De tal modo, se conformó una muestra total de 512 estudiantes reunidos en dos grupos balanceados de consumidores de sustancias ilegales y no consumidores, con los que se llevaron a cabo los análisis comparativos y predictivos correspondientes (Figura 1).

\section{Instrumento}

Se aplicó un cuestionario diseñado ex profeso para evaluar las cinco competencias socioemocionales anteriormente mencionadas: regulación emocional, toma responsable y reflexiva de decisiones, establecimiento de relaciones positivas con pares, control conductual y capacidad de ajuste escolar (CIJ, 2017). El formato de respuesta fue tipo Likert con cuatro opciones de frecuencia (con puntuaciones entre cero y cuatro). El consumo de sustancias se evaluó mediante ocho reactivos referentes al consumo de mariguana, inhalables, cocaína y otras sustancias, alguna vez en la vida.

El contenido de los factores relativos a las competencias socioemocionales estudiadas, tal como quedaron 


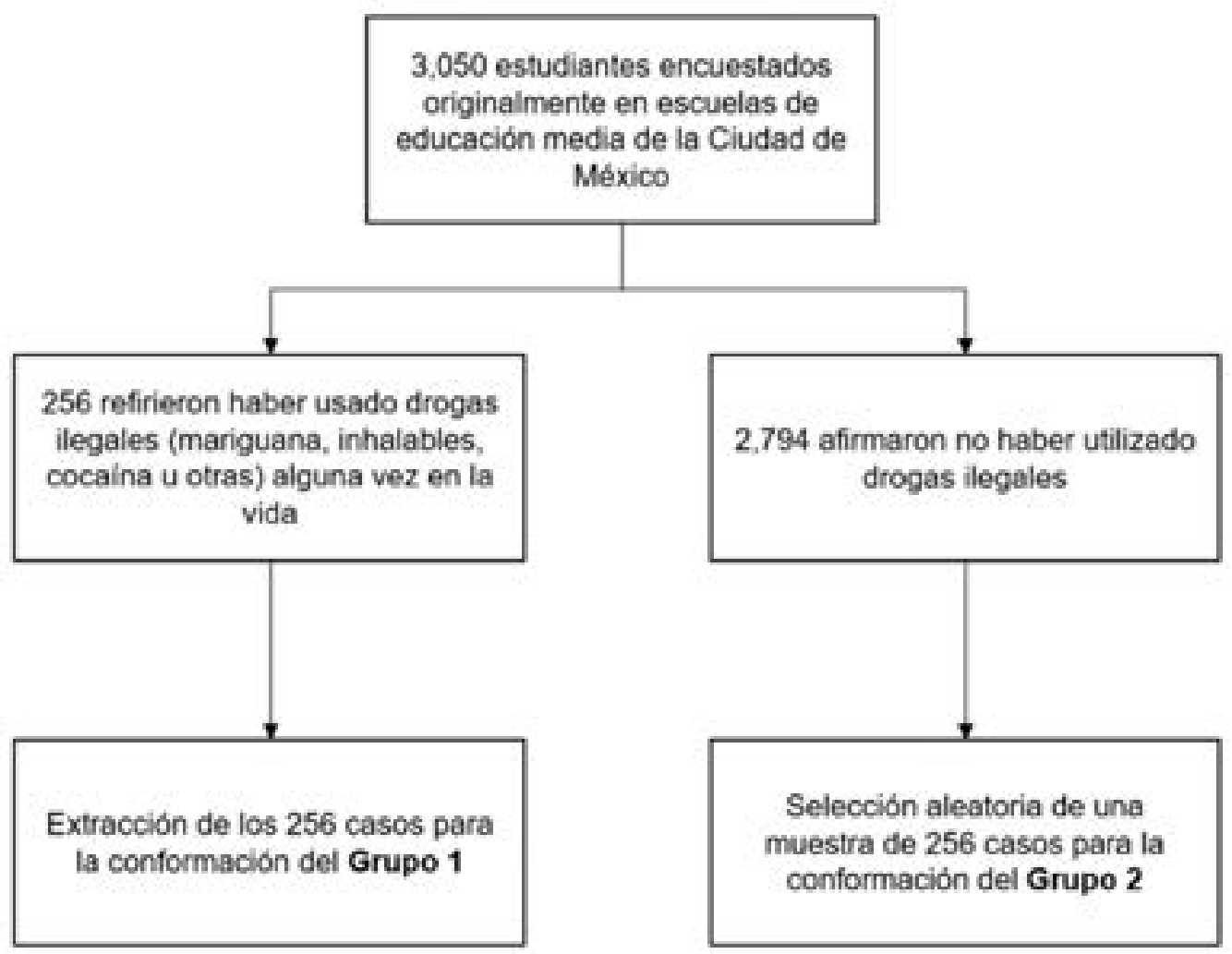

Figura 1. Conformación de la muestra

integrados a partir de su validación, se describe a continuación:

- Inadecuada regulación emocional: se refiere a la dificultad para regular las propias emociones, así como a la cualidad perturbadora de ciertos afectos experimentados.

- Bajo control conductual: explora la frecuencia de comportamientos impulsivos y explosivos, así como la susceptibilidad a la influencia de pares.

- Toma reflexiva de decisiones: evalúa la habilidad para identificar las consecuencias de la propia conducta, sopesar distintos cursos de acción y tomar decisiones responsables (para efectos del análisis de riesgos, las puntuaciones de este factor fueron invertidas).

- Capacidad para entablar relaciones positivas con pares: se refiere a la capacidad para establecer y mantener relaciones interpersonales basadas en la aceptación y la confianza, así como para ofrecer y recibir apoyo (en el análisis, las puntuaciones fueron igualmente invertidas a fin de contar con indicadores de una baja capacidad de integración a la comunidad escolar como presunto factor de riesgo).
- Capacidad de ajuste escolar: explora la motivación por el estudio, el disfrute y sentido del logro asociados a la participación en actividades escolares y la capacidad para responder a las demandas del propio medio escolar (las calificaciones fueron transformadas para indicar, en el análisis de riesgos, una baja capacidad de ajuste).

El instrumento fue previamente validado y mostró adecuadas propiedades psicométricas para su uso en población estudiantil mexicana (CIJ, 2017). El análisis factorial mostró la solución de cinco factores descritos, bien diferenciados, con una $X^{2}=32158.034$ y $p=.000$, explicando el $49 \%$ de la varianza. La fiabilidad para el instrumento general fue de 0.89 , en tanto que la de las cinco escalas que lo conforman se situó por arriba de .8.

\section{Procedimiento}

La aplicación del cuestionario estuvo a cargo de los propios investigadores, apoyados por un equipo de cuatro estudiantes de Psicología en servicio social. Para la habilitación de este equipo, se elaboró una guía de aplica- 
ción y se efectuó una capacitación práctica, incluyendo ejercicios de roles dirigidos a perfeccionar los procedimientos y garantizar su homogeneidad.

\section{Análisis}

Se realizó en primera instancia un análisis descriptivo de variables. Posteriormente, para comparar las puntuaciones medias obtenidas por los dos grupos de estudio en las escalas de competencias socioemocionales, se aplicó una prueba $t$ de Student para muestras independientes con intervalo de confianza al 95\%. Para una adecuada interpretación de los resultados, se atendió a la prueba de Levene para la homogeneidad de varianzas.

Por último, se realizó un análisis de regresión logística con el método Introducir en un solo bloque, dirigido a estimar la capacidad predictiva y explicativa del modelo conformado por los factores de baja competencia socioemocional, en tanto variables predictoras del uso experimental de sustancias (uso alguna vez en la vida) como variable criterio.

Se calcularon las pruebas Ómnibus -estadísticos de contraste que permiten validar la posibilidad de llevar a cabo una modelación probabilística, identificando la plausibilidad del modelo propuesto- y la prueba de Hosmer y Lemeshow, que evalúa la bondad de ajuste. Establecida la validez inicial del modelo, se estimó, en seguida, el poder de clasificación de la ecuación propuesta y se calculó el coeficiente de determinación $R^{2}$ de Nagelkerke que indica la proporción de varianza de la variable criterio explicada por el modelo. El coeficiente de regresión correspondiente a cada variable predictora se reporta incluyendo el estadístico Wald para evaluar la hipótesis nula, el nivel de significancia y el intervalo de confianza al 95\%.

\section{Consideraciones éticas}

El trato otorgado a los estudiantes participantes en la encuesta cumplió, en todo momento, con las medidas requeridas de cuidado ético. Se obtuvo la autorización informada de autoridades escolares, el consentimiento de padres o tutores y el "asentimiento" de los propios alumnos; se garantizó sistemáticamente el anonimato de los participantes y la confidencialidad de la información personal proporcionada.

\section{RESULTADOS}

\section{Participantes}

El grupo de usuarios de drogas $(n=256)$ registró una edad promedio de 12 años $(D E=.5)$ y una distribución por sexo de $57.6 \%$ hombres $(n=147)$ y $42.4 \%$ mujeres
( $n=108)$; mientras que el grupo de no usuarios $(n=256)$ reportó una edad de 11.98 años $(D E=.4)$, con una distribución de $56.3 \%$ hombres $(n=142)$ y $43.7 \%$ mujeres $(n=110)$. Estas diferencias entre grupos no resultaron estadísticamente significativas.

\section{Distribución de calificaciones de competencia so- cioemocional}

En general, se registraron calificaciones que indican un nivel de riesgo intermedio. Las puntuaciones más elevadas correspondieron a un bajo control conductual, con una media de $2.65(D E=.93)$, seguido por dificultades para entablar relaciones positivas con pares, con una media de $2.32(D E=.71)$, irreflexiva toma de decisiones, con un promedio de $2.16(D E=.71)$, deficiente ajuste escolar, con media de $2.1(D E=.63) \mathrm{y}$, finalmente, inadecuada regulación emocional, con una media de $2.06(D E=.6)$.

\section{Análisis comparativo}

En la comparación de las medias registradas por ambos grupos de estudio se encontraron diferencias estadísticamente significativas; el grupo de consumidores obtuvo calificaciones de déficit o inadecuación socioemocional más elevadas (Tabla 1), con diferencias mayores en las escalas de inadecuada regulación emocional, ineficiente ajuste escolar y bajo control conductual.

\section{Análisis de predictores}

Inicialmente, el análisis de regresión efectuado para determinar la capacidad predictiva del modelo de bajas competencias socioemocionales arrojó resultados de adecuación muestral y bondad de ajuste significativos. En cuanto a la clasificación de casos obtenida con la ecuación propuesta, se registró una sensibilidad de $71 \%$ y una especificidad de $66.5 \%$, es decir, una clasificación adecuada del $68.8 \%$ de la muestra, lo cual puede considerarse aceptable. Por su parte, el coeficiente de determinación $R^{2}$ indica una proporción de varianza explicada de $21.5 \%$.

Como puede apreciarse en la Tabla 2, teniendo en cuenta el efecto conjunto de todas las variables incluidas en el modelo, una deficiente regulación emocional, un inadecuado ajuste escolar y un bajo control conductual resultaron predictores significativos del consumo de drogas alguna vez en la vida, representando un riesgo 155,78 y $40 \%$ mayor, respectivamente.

\section{DISCUSIÓN}

Los resultados del presente estudio concuerdan, en líneas generales, con lo reportado por la investigación de 
Tabla 1

Comparación de medias obtenidas en la escala de competencias socioemocionales en estudiantes de primer grado de educación media, usuarios y no usuarios de drogas ( $n=256$ por grupo)

\begin{tabular}{|c|c|c|c|c|c|}
\hline $\begin{array}{c}\text { Competencias } \\
\text { socioemocionales }\end{array}$ & Grupo & Media & $\mathrm{DE}$ & $t$ & Sig. \\
\hline \multirow{2}{*}{ Inadecuada regulación emocional } & Usuarios & 2.25 & .61 & \multirow{2}{*}{7.327} & \multirow{2}{*}{.000} \\
\hline & No usuarios & 1.88 & .54 & & \\
\hline \multirow{2}{*}{ Dificultad de ajuste escolar } & Usuarios & 2.26 & .64 & \multirow{2}{*}{5.622} & \multirow{2}{*}{.000} \\
\hline & No usuarios & 1.95 & .59 & & \\
\hline \multirow{2}{*}{ Bajo control conductual } & Usuarios & 2.87 & .85 & \multirow{2}{*}{5.615} & \multirow{2}{*}{.001} \\
\hline & No usuarios & 2.42 & .97 & & \\
\hline \multirow{2}{*}{$\begin{array}{l}\text { Dificultad para entablar } \\
\text { relaciones positivas } \\
\text { con pares }\end{array}$} & Usuarios & 2.42 & .75 & \multirow[b]{2}{*}{3.200} & \multirow[b]{2}{*}{.000} \\
\hline & No usuarios & 2.22 & .66 & & \\
\hline \multirow{2}{*}{ Toma irreflexiva de decisiones } & Usuarios & 2.23 & .74 & \multirow{2}{*}{2.333} & \multirow{2}{*}{.020} \\
\hline & No usuarios & 2.90 & .68 & & \\
\hline
\end{tabular}

Tabla 2

Competencias socioemocionales como predictores del uso de drogas ilícitas alguna vez en la vida en estudiantes de primer grado de educación media

Regresión logística, método Introducir $(n=512)$

\begin{tabular}{ccccc}
\hline $\begin{array}{c}\text { Competencias } \\
\text { socioemocionales }\end{array}$ & Wald & Sig. & RM & IC 95\% \\
\hline Inadecuada regulación emocional & 27.682 & .000 & 2.551 & $1.800-3.615$ \\
Dificultad de ajuste escolar & 8.978 & .003 & 1.779 & $1.221-2.594$ \\
Bajo control conductual & 8.860 & .003 & 1.403 & $1.123-1.753$ \\
Dificultad para entablar relaciones & 1.422 & .233 & 1.236 & $.872-1.751$ \\
positivas con pares & .074 & .786 & .956 & $.692-1.321$ \\
Toma irreflexiva de decisiones & & &
\end{tabular}

factores de riesgo del uso de drogas, al tiempo que permiten jerarquizar el efecto predictor de una inadecuada regulación emocional, un ajuste escolar deficiente y un bajo control conductual.

En primer lugar, los hallazgos coinciden con los estudios que identifican a un bajo nivel de inteligencia y de regulación emocionales como factores predictores del consumo de sustancias (Claros \& Sharma, 2010; Fernández-Serrano et al., 2012; Ferreira, et al., 2012; Hessler \& Fainsilber, 2010; Hill \& Maggi, 2011; Law \& Yan, 2011; Sharma et al., 2017).

Destaca, asimismo, la relevancia de una menor capacidad de ajuste escolar, en específico de una baja motivación por el estudio y deficiente desempeño académico, en coincidencia con aquellos autores que han subraya- do el papel de la adherencia escolar como factor que reduce el riesgo del consumo de sustancias (Arillo-Santillán et al., 2002; ClJ, 1999; Díaz \& García-Aurrecoechea, 2008; Hawkings et al., 1992; Kandel, 1985; Smart et al., 1981; Smith \& Fogg, 1978; Villarreal-González et al., 2010).

Respecto al efecto de un bajo control conductual, se confirma la investigación que ha reportado una alta correlación entre consumo e impulsividad (De Witt, 2009; Díaz \& García-Aurrecoechea, 2008; Díaz \& Moral, 2018; Pedrero, 2007; Perea-Baena \& Oña-Compan, 2011; Wills et al., 2011).

Al considerar el carácter complejo y multideterminado del uso de drogas, cabe señalar que el valor explicativo del modelo presentado resulta aceptable, lo que supone 
que efectivamente puede guiar el diseño de contenidos de programas dirigidos a la prevención del uso de sustancias con jóvenes estudiantes.

Entre las limitaciones del estudio se puede destacar que la recolección de información fue de carácter transversal, de modo que los hallazgos obtenidos requerirían ser objeto de corroboración mediante diseños de investigación longitudinales. Por otro lado, el modelo de competencias evaluado en este artículo podría ser enriquecido mediante la incorporación de competencias planteadas en otros modelos (CASEL, 2015; Lerner et al., 2009; Lerner, 2004; SEP/PNUD, 2018; Travis \& Leech, 2014). Por último, convendría ampliar el estudio de las competencias socioemocionales en su relación con el uso de drogas en otros grupos poblacionales, principalmente de jóvenes de mayor y menor edad o no escolarizados, entre otros.

\section{FUENTES DE FINANCIAMIENTO}

Este estudio no contó con fuentes de financiamiento.

\section{CONFLICTOS DE INTERÉS}

Los autores manifiestan que no existe ningún conflicto de interés en la realización del manuscrito.

\section{REFERENCIAS}

Arillo-Santillán, E., Fernández, E., Hernández-Ávila, M., Tapia-Uribe, M., Cruz-Valdés, A., \& Lazcano-Ponce, E. C. (2002). Prevalencia de tabaquismo y bajo desempeño escolar, en estudiantes de 11 a 24 años de edad del estado de Morelos, México. Salud Pública de México, 44(Suplemento 1), S54-S66.

Bar-On, R. (2006). The Bar-On model of emotional-social intelligence (ESI). Psicothema, 18(Supl), 13-25.

Blair, M. A., Stewart, J. L., May, A. C., Rezke, M., Tapert, S. F., \& Paulus, M. P. (2018). Blunted frontostriatal blood oxygen level-dependent signals predict stimulant and marijuana use. Biological Psychiatry: Cognitive Neuroscience and Neuroimaging. doi: 10.1016/ j.bpsc.2018.03.005

Carpenter, K. M., Bedi, G., \& Vadhan, N. P. (2015). Understanding and shifting drug-related decisions: Contributions of automatic decision-making processes. Current Psychiatry Reports, 17(8), 1-11. doi:10.1007/s11920-015-0607-8

Centros de Integración Juvenil. (1999). Adherencia escolar y consumo de drogas. Informe de Investigación 99-07. México: Centros de Integración Juvenil, A.C.

Centros de Integración Juvenil. (2015). Sistema Institucional de Evaluación de Programas de Prevención, Evaluación de resultados del programa con estudiantes de educación media, Tercera fase, Informe global. Informe de Investigación 15-11. México: Centros de Integración Juvenil, A.C.
Centros de Integración Juvenil. (2016). Guía Técnica del Programa Preventivo "Para Vivir sin Adicciones". México: Centros de Integración Juvenil, A.C.

Centros de Integración Juvenil. (2017). Evaluación del Programa Preventivo 2017-2020, línea base. Informe de Investigación 1017. México: Centros de Integración Juvenil, A.C.

Claros, E., \& Sharma, M. (2010). The relationship between emotional intelligence and abuse of alcohol, marijuana, and tobacco among college students. Journal of Alcohol and Drug Education, 56(1), 8-37.

Collaborative for Academic, Social, and Emotional Learning [CASEL], 2015. Guide: Effective Social and Emotional Learning Programs-Middle and High School Edition. Illinois: autor.

De Witt, H. (2009). Impulsivity as a determinant and consequence of drug use: A review of underlying processes. Addiction Biology, 14(1), 22-31. doi: 10.1111/j.1369-1600.2008.00129.x.

Díaz, B. A. (2006). El enfoque de competencias en la educación. ¿Una alternativa o un disfraz de cambio? Perfiles Educativos, 23(11), 7-36

Díaz, N. B., \& García-Aurrecoechea, R. (2008). Factores psicosociales de riesgo de consumo de drogas ilícitas en una muestra de estudiantes mexicanos de educación media. Revista Panamericana de Salud Pública, 24(4), 223-232.

Díaz, G. N., \& Moral, J.M. (2018). Consumo de alcohol y conducta antisocial e impulsividad en adolescentes españoles. Acta Colombiana de Psicología, 21(2), 110-130. doi: 10.14718/ACP. 2018.21.2.6

Ekhtiari, H., Víctor, T. A., \& Paulus, M. P. (2016). Aberrant decision-making and drug addiction - how strong is the evidence? Current Opinion in Behavioral Sciences, 13, 25-33. doi: 10.1016/ j.cobeha.2016.09.002

Fernández-Serrano, M. J., Moreno-López, L., Pérez-García, M., \& Verdejo-García, A. (2012). Inteligencia emocional en individuos dependientes de cocaína. Trastornos Adictivos, 14(1), 1-33.

Ferreira, M., Simoes, C., Gaspar, M. M., Ramiro, L., \& Alves, D. J. (2012). The role of social and emotional competence on risk behaviors in adolescence. The International Journal of Emotional Education, 4(1), 43-55.

Gardner, H. (1983). Estructuras de la mente. La teoría de las inteligencias múltiples. Nueva York: Harper Collins Publisher Inc.

Goleman, D. (2001). Inteligencia Emocional. España: Editorial Kairós.

Hawkings, D., Catalano, R., \& Miller, J. (1992). Risk and protective factors for alcohol and other drug problems in adolescence and early adulthood: implications for substance abuse prevention. Psychological Bulletin, 112(1), 64-105.

Hessler, D., \& Fainsilber, K. L. (2010). Brief Report: Associations between emotional competence and adolescent risky behavior Journal of Adolescent Health, 33(1), 241-260. doi: 10.1016/j. adolescence.2009.04.007

Hill, E., \& Maggi, S. (2011). Emotional intelligence and smoking: protective and risk factors among Canadian young adults. Personality and Individual Differences, 51(1), 45-50. doi: 10.1016/j. paid.2011.03.008 
Instituto Nacional de Psiquiatría Ramón de la Fuente Muñiz; Comisión Nacional Contra las Adicciones y Secretaría de Salud (2015). Encuesta Nacional de Consumo de Drogas en Estudiantes 2014. Reportes Drogas, Tabaco y Alcohol. México DF, México: INPRFM.

Jonnaert, P., Barrette, J., Masciotra, D., \& Yaya, M. (2006). Revisión de la competencia como organizadora de los programas de formación: hacia un desempeño competente. Observatorio de Reformas educativas. Ginebra: Oficina Internacional de educación/ BIE/UNESCO. Disponible: http://www.ibe.unesco.org/fileadmin /user_upload/COPs/Pages_documents/Competencies/ORE_ Spanish.pdf

Kandel, D. B. (1985). On processes of peer influences in adolescent drug use: A developmental perspective. Advances in Alcohol \& Substance Abuse, 4(3-4), 139-163. doi: 10.1300/J251v04n03-07

Law, B. M. F., \& Yan, L. T. (2011). Importance of emotional competence in designing an antidrug education curriculum for junior secondary school students in Hong Kong. The Scientific World Journal 11, 2257-2265. doi:10.1100/2011/127894

Lerner, R. M. (2004). The SAGE Program on Applied Developmental Science: Liberty: Thriving and civic engagement among America's youth. Thousand Oaks, CA: SAGE Publications, Inc. doi: 10.4135/9781452233581

Lerner, J. V., Phelps, E., Forman, Y., \& Bowers, E. P. (2009). Positive youth development. In Lerner, R.M. \& Steinberg, L. (Eds.), Handbook of adolescent psychology: Vol 1. Individual bases of adolescent development (3rd ed., pp. 524-558). Hoboken, Nueva Jersey: Wiley.

López-Zafra, E., Pulido, M., \& Berrios, P. (2014). EQI version corta (EQI-C), Adaptación y validación al español del EQ-i en universitarios. Boletín de Psicología 110, 21-36.

Manrique-Abril, F. G., Ospina, J. M., \& Garcia-Ubaque J. C. (2009). Consumo de alcohol y tabaco en escolares y adolescentes de Tunja, Colombia, 2009. Revista de Salud Pública, 13(1), 89-101.

Mata, F. G., Neves, F. S., Lage, G. M., Moraes, P. H., Mattos, P., Fuentes, D., Correa, H., \& Malloy, D. L. (2011). Neuropsychological assessment of the decision making process in children and adolescents: an integrative review of the literature. Archives of Clinical Psychiatry, 38(3), 106-115. doi: 10.1590/S010160832011000300005

Pedrero, E. J. (2007).Comportamientos adictivos y déficit de control de impulsos. Revista Española de Drogodependencias, 32(4), 488-511.

Peñafiel, E. (2009). Factores de riesgo y protección en el consumo de sustancias en adolescentes. Pulso 32, 174-173.
Perea-Baena, J. M., \& Oña-Compan, S. (2011). Impulsividad como predictor de recaída en el abandono de tabaco. Anales de Psicología, 27(1), 1-6.

Pérez-Islas, V., \& Arellanez-Hernández, J. L. (2015). Vínculo afectivo con padres, pares y uso de drogas en adolescentes mexicanos. Revista de Sistemas Experimentales, 2(5), 178-192.

Rodríguez, S., Pérez, V., \& Córdova-Alcaráz, A. (2007). Factores familiares y de pares asociados al consumo de drogas en estudiantes de educación media, Revista Intercontinental de Psicología y Educación, 9(1), 159-186.

Salovey, P., \& Mayer, J. D. (1990). Emotional intelligence. Imagination, Cognition, and Personality, 9(3), 185-211. doi: 10.2190/DUGGP24E-52WK-6CDG

Secretaría de Educación Pública, Programa de las Naciones Unidas para el Desarrollo (2018). Construye T. Programa de la Secretaría de Educación Pública y el Programa de las Naciones Unidas para el Desarrollo. Disponible: http://www.construye-t.org.mx/

Sharma, D., Gulati, R., \& Misra, I. (2017). Emotional intelligence: influencing smoking behavior in young adults. Jindal Journal of Business Research, 6(1), 14-24.

Smart, R., Medina-Mora, M. E., Terraoba, G., \& Varma, V. K. (1981). Drug use among non-students in three countries. Drug and Alcohol Dependence, 7(2), 125-132. doi: 10.1016/0376-8716(81)90026-0

Smith, G. M., \& Fogg, C. P. (1978). Psychological predictors of early use, late use, and non use of marijuana among teenage student. En D. Kendel (1978). Longitudinal research on drug use: Empirical findings and methodological issues. Washington, D.C.: Hemisphere Wiley.

Sussman, S., Earleywine, M., Wills, T., Cody, C., Bigdan, T., Dent, C., \& Newcomb, M. D. (2016). The motivation, skills and decision-making model of "drug abuse" prevention. Substance Use \& Misuse, 39(10-12), 1971-2016. doi: 10.1081/LSUM-200034769

Travis, R., \& Leech, T. (2014). Empowerment-based positive youth developement: A new understanding of healthy developement for african-american youth. Journal of Research on Adolescence, 24(1), 1-24. doi: 10.1111/jora.12062

Villarreal-González, M. E., Sánchez-Sosa, J. C., Musitu, G., \& Varela, R. (2010). El consumo de alcohol en adolescentes escolarizados: propuesta de un modelo sociocomunitario. Intervención Psicosocial, 3(19), 253-264.

Wills, T. A., Pokhrel, P., Morehouse, E., \& Fenster, B. (2011) Behavioral and emotional regulation and adolescents substance use problems: a test of moderation effects in a dual-process model. Psychology of Addictive Behaviors, 25(2), 279-292. doi: 10.1037/a0022870 